\title{
Ceramide turnover (version 2020.5) in the IUPHAR/BPS Guide to Pharmacology Database
}

\author{
Anthony H. Futerman ${ }^{1}$ \\ 1. Weizmann Institute of Science, Israel
}

\begin{abstract}
Ceramides are a family of sphingophospholipids synthesized in the endoplasmic reticulum, which mediate cell stress responses, including apoptosis, autophagy and senescence, Serine palmitoyltransferase generates 3ketosphinganine, which is reduced to dihydrosphingosine (dihydrosphingosine). N-Acylation allows the formation of dihydroceramides, which are subsequently reduced to form ceramides. Once synthesized, ceramides are trafficked from the ER to the Golgi bound to the ceramide transfer protein, CERT (COL4A3BP, Q9Y5P4). Ceramide can be metabolized via multiple routes, ensuring tight regulation of its cellular levels. Addition of phosphocholine generates sphingomyelin while carbohydrate is added to form glucosyl- or galactosylceramides. Ceramidase re-forms sphingosine or sphinganine from ceramide or dihydroceramide. Phosphorylation of ceramide generates ceramide phosphate. The determination of accurate kinetic parameters for many of the enzymes in the sphingolipid metabolic pathway is complicated by the lipophilic nature of the substrates.
\end{abstract}

\section{Contents}

This is a citation summary for Ceramide turnover in the Guide to Pharmacology database (GtoPdb). It exists purely as an adjunct to the database to facilitate the recognition of citations to and from the database by citation analyzers. Readers will almost certainly want to visit the relevant sections of the database which are given here under database links.

GtoPdb is an expert-driven guide to pharmacological targets and the substances that act on them. GtoPdb is a reference work which is most usefully represented as an on-line database. As in any publication this work should be appropriately cited, and the papers it cites should also be recognized. This document provides a citation for the relevant parts of the database, and also provides a reference list for the research cited by those parts.

Please note that the database version for the citations given in GtoPdb are to the most recent preceding version in which the family or its subfamilies and targets were substantially changed. The links below are to the current version. If you need to consult the cited version, rather than the most recent version, please contact the GtoPdb curators.

\section{Database links}

Ceramide turnover

https://www.guidetopharmacology.org/GRAC/FamilyDisplayForward?familyld=767

Serine palmitoyltransferase

https://www.guidetopharmacology.org/GRAC/FamilyDisplayForward?familyld=788

Enzymes

SPT1(serine palmitoyltransferase long chain base subunit 1)

https://www. guidetopharmacology.org/GRAC/ObjectDisplayForward?objectld=2509

SPT2(serine palmitoyltransferase long chain base subunit 2)

https://www. guidetopharmacology.org/GRAC/ObjectDisplayForward?objectld=2510

SPT3(serine palmitoyltransferase long chain base subunit 3)

https://www. guidetopharmacology.org/GRAC/ObjectDisplayForward?objectld=2511

SPTSSA(serine palmitoyltransferase small subunit A)

https://www.guidetopharmacology.org/GRAC/ObjectDisplayForward?objectld=2512

SPTSSB(serine palmitoyltransferase small subunit B)

https://www. guidetopharmacology.org/GRAC/ObjectDisplayForward?objectld=2513

3-ketodihydrosphingosine reductase

https://www.guidetopharmacology.org/GRAC/FamilyDisplayForward?familyld=791 


\section{Enzymes}

3-ketodihydrosphingosine reductase

https://www.guidetopharmacology.org/GRAC/ObjectDisplayForward?.objectld=2463

Ceramide synthase

https://www.guidetopharmacology.org/GRAC/FamilyDisplayForward?familyld=789

Enzymes

CERS1(ceramide synthase 1)

https://www.guidetopharmacology.org/GRAC/ObjectDisplayForward?objectld=2474

CERS2(ceramide synthase 2)

https://www. guidetopharmacology.org/GRAC/ObjectDisplayForward? objectld=2475 CERS3(ceramide synthase 3)

https://www.guidetopharmacology.org/GRAC/ObjectDisplayForward?objectld=2476 CERS4(ceramide synthase 4)

https://www. guidetopharmacology.org/GRAC/ObjectDisplayForward?objectld=2477 CERS5(ceramide synthase 5)

https://www. guidetopharmacology.org/GRAC/ObjectDisplayForward?objectld=2478 CERS6(ceramide synthase 6)

https://www.guidetopharmacology.org/GRAC/ObjectDisplayForward?objectld=2479

Sphingolipid $\Delta^{4}$-desaturase

https://www.guidetopharmacology.org/GRAC/FamilyDisplayForward?familyld=790

Enzymes

delta 4-desaturase, sphingolipid 1

https://www. guidetopharmacology.org/GRAC/ObjectDisplayForward?objectld=2484

delta 4-desaturase, sphingolipid 2

https://www. guidetopharmacology.org/GRAC/ObjectDisplayForward?objectld=2485

Sphingomyelin synthase

https://www. guidetopharmacology.org/GRAC/FamilyDisplayForward?familyld=774

Introduction to Sphingomyelin synthase

https://www. guidetopharmacology.org/GRAC/FamilylntroductionForward?familyld=774

Enzymes

sphingomyelin synthase 1

https://www.guidetopharmacology.org/GRAC/ObjectDisplayForward?objectld=2520 sphingomyelin synthase 2

https://www.guidetopharmacology.org/GRAC/ObjectDisplayForward?objectld=2521

sterile alpha motif domain containing 8

https://www.guidetopharmacology.org/GRAC/ObjectDisplayForward?objectld=2525

Sphingomyelin phosphodiesterase

https://www.guidetopharmacology.org/GRAC/FamilyDisplayForward?familyld=773

Enzymes

sphingomyelin phosphodiesterase 1

https://www. guidetopharmacology.org/GRAC/ObjectDisplayForward?objectld=2514 sphingomyelin phosphodiesterase 2

https://www.guidetopharmacology.org/GRAC/ObjectDisplayForward?objectld=2515 sphingomyelin phosphodiesterase 3

https://www. guidetopharmacology.org/GRAC/ObjectDisplayForward?objectld=2516 sphingomyelin phosphodiesterase 4

https://www.guidetopharmacology.org/GRAC/ObjectDisplayForward?objectld=2517 sphingomyelin phosphodiesterase acid-like 3A

https://www. guidetopharmacology.org/GRAC/ObjectDisplayForward?objectld=2518 sphingomyelin phosphodiesterase acid-like 3B

https://www. guidetopharmacology.org/GRAC/ObjectDisplayForward?objectld=2519

Neutral sphingomyelinase coupling factors

https://www. guidetopharmacology.org/GRAC/FamilyDisplayForward?familyld=772

Enzymes

embryonic ectoderm development

https://www.guidetopharmacology.org/GRAC/ObjectDisplayForward?objectld=2487 neutral sphingomyelinase activation associated factor

https://www. guidetopharmacology.org/GRAC/ObjectDisplayForward?objectld=2495

Ceramide glucosyltransferase

https://www. guidetopharmacology.org/GRAC/FamilyDisplayForward?familyld=775

Enzymes

UDP-glucose ceramide glucosyltransferase

https://www.guidetopharmacology.org/GRAC/ObjectDisplayForward?objectld=2528

Acid ceramidase

https://www.guidetopharmacology.org/GRAC/FamilyDisplayForward?familyld=769

Enzymes

$\mathrm{N}$-acylsphingosine amidohydrolase 1 
https://www.guidetopharmacology.org/GRAC/ObjectDisplayForward?objectld=2491

Neutral ceramidases

https://www. guidetopharmacology.org/GRAC/FamilyDisplayForward?familyld=770

Enzymes

$\mathrm{N}$-acylsphingosine amidohydrolase 2

https://www.guidetopharmacology.org/GRAC/ObjectDisplayForward?objectld=2492

$\mathrm{N}$-acylsphingosine amidohydrolase 2B

https://www.guidetopharmacology.org/GRAC/ObjectDisplayForward?objectld=2493

Alkaline ceramidases

https://www.guidetopharmacology.org/GRAC/FamilyDisplayForward?familyld=768

Enzymes

alkaline ceramidase 1

https://www. guidetopharmacology.org/GRAC/ObjectDisplayForward?objectld=2468

alkaline ceramidase 2

https://www. guidetopharmacology.org/GRAC/ObjectDisplayForward?objectld=2469

alkaline ceramidase 3

https://www.guidetopharmacology.org/GRAC/ObjectDisplayForward?objectld=2470

Ceramide kinase

https://www.guidetopharmacology.org/GRAC/FamilyDisplayForward?familyld=771

Enzymes

ceramide kinase

https://www.guidetopharmacology.org/GRAC/ObjectDisplayForward?objectld=2473

\section{References}

1. Adam-Klages S, Adam D, Wiegmann K, Struve S, Kolanus W, Schneider-Mergener J and Krönke M. (1996) FAN, a novel WD-repeat protein, couples the p55 TNF-receptor to neutral sphingomyelinase. Cell 86: 937-47 [PMID:8808629]

2. Beauchamp E, Tekpli X, Marteil G, Lagadic-Gossmann D, Legrand P and Rioux V. (2009) N-Myristoylation targets dihydroceramide Delta4-desaturase 1 to mitochondria: partial involvement in the apoptotic effect of myristic acid. Biochimie 91: 1411-9 [PMID:19647031]

3. Butters TD, van den Broek LAGM, Fleet GWJ, Krulle TM, Wormald MR, Dwek RA and Platt FM. (2000) Molecular requirements of imino sugars for the selective control of $\mathrm{N}$-linked glycosylation and glycosphingolipid biosynthesis. Tetrahedron: Assymetry 11: 113-124

4. Camacho L, Simbari F, Garrido M, Abad JL, Casas J, Delgado A and Fabriàs G. (2012) 3-Deoxy-3,4dehydro analogs of XM462. Preparation and activity on sphingolipid metabolism and cell fate. Bioorg. Med. Chem. 20: 3173-9 [PMID:22537678]

5. Chan HM, Gu X-JJ, Huang Y, Li L, Mi Y, Qi W, Sendzik M, Sun Y, Wang L and Yu Zet al.. (2016) Triazolopyrimidine compounds and uses thereof Patent number: WO2016103155A1.

6. Chen Y and Cao Y. (2017) The sphingomyelin synthase family: proteins, diseases, and inhibitors.Biol. Chem. 398: 1319-1325 [PMID:28742512]

7. Cingolani F, Casasampere M, Sanllehí P, Casas J, Bujons J and Fabrias G. (2014) Inhibition of dihydroceramide desaturase activity by the sphingosine kinase inhibitor SKI II. J. Lipid Res. 55: 1711-20 [PMID:24875537]

8. Coant N, Sakamoto W, Mao C and Hannun YA. (2017) Ceramidases, roles in sphingolipid metabolism and in health and disease. Adv Biol Regul 63: 122-131 [PMID:27771292]

9. Deng X, Lin F, Zhang Y, Li Y, Zhou L, Lou B, Li Y, Dong J, Ding T and Jiang Xt al.. (2014) Identification of small molecule sphingomyelin synthase inhibitors. Eur J Med Chem 73: 1-7 [PMID:24374347]

10. Fabrias G, Muñoz-Olaya J, Cingolani F, Signorelli P, Casas J, Gagliostro V and Ghidoni R. (2012) Dihydroceramide desaturase and dihydrosphingolipids: debutant players in the sphingolipid arena. Prog. Lipid Res. 51: 82-94 [PMID:22200621]

11. Graf C, Klumpp M, Habig M, Rovina P, Billich A, Baumruker T, Oberhauser B and Bornancin F. (2008) Targeting ceramide metabolism with a potent and specific ceramide kinase inhibitor. Mol. Pharmacol. 74: 925-32 [PMID:18612076]

12. Han G, Gupta SD, Gable K, Niranjanakumari S, Moitra P, Eichler F, Brown Jr RH, Harmon JM and Dunn TM. (2009) Identification of small subunits of mammalian serine palmitoyltransferase that confer distinct acyl-CoA substrate specificities. Proc. Natl. Acad. Sci. U.S.A. 106: 8186-91 [PMID:19416851]

13. He Y, Selvaraju S, Curtin ML, Jakob CG, Zhu H, Comess KM, Shaw B, The J, Lima-Fernandes E and Szewczyk MM et al.. (2017) The EED protein-protein interaction inhibitor A-395 inactivates the PRC2 complex. Nat. Chem. Biol. 13: 389-395 [PMID:28135237]

14. Hoch DG, Abegg D, Hannich JT, Pechalrieu D, Shuster A, Dwyer BG, Wang C, Zhang X, You Q and Riezman H et al.. (2020) Combined Omics Approach Identifies Gambogic Acid and Related Xanthones as Covalent Inhibitors of the Serine Palmitoyltransferase Complex. Cell Chem Biol 27: 586-597.e12 [PMID:32330443]

15. Houben E, Holleran WM, Yaginuma T, Mao C, Obeid LM, Rogiers V, Takagi Y, Elias PM and Uchida Y. (2006) Differentiation-associated expression of ceramidase isoforms in cultured keratinocytes and epidermis. J. Lipid Res. 47: 1063-70 [PMID:16477081] 
16. Jiang X-C, Yeang C, Li Z, Chakraborty M, Zhang H and Fan Y.. (2009) Sphingomyelin biosynthesis: its impact on lipid metabolism and atherosclerosis. Clinical Lipidology 4: 595-609

17. Koch J, Gärtner S, Li CM, Quintern LE, Bernardo K, Levran O, Schnabel D, Desnick RJ, Schuchman EH and Sandhoff K. (1996) Molecular cloning and characterization of a full-length complementary DNA encoding human acid ceramidase. Identification Of the first molecular lesion causing Farber disease. J. Biol. Chem. 271: 33110-5 [PMID:8955159]

18. Lahiri S and Futerman AH. (2005) LASS5 is a bona fide dihydroceramide synthase that selectively utilizes palmitoyl-CoA as acyl donor. J. Biol. Chem. 280: 33735-8 [PMID:16100120]

19. Laviad EL, Albee L, Pankova-Kholmyansky I, Epstein S, Park H, Merrill Jr AH and Futerman AH. (2008) Characterization of ceramide synthase 2: tissue distribution, substrate specificity, and inhibition by sphingosine 1-phosphate. J. Biol. Chem. 283: 5677-84 [PMID:18165233]

20. Li YL, Qi XY, Jiang H, Deng XD, Dong YP, Ding TB, Zhou L, Men P, Chu Y and Wang RXet al.. (2015) Discovery, synthesis and biological evaluation of 2-(4-(N-phenethylsulfamoyl)phenoxy)acetamides (SAPAs) as novel sphingomyelin synthase 1 inhibitors. Bioorg. Med. Chem. 23: 6173-84 [PMID:26314925]

21. Li Z, Fan Y, Liu J, Li Y, Huan C, Bui HH, Kuo MS, Park TS, Cao G and Jiang XC. (2012) Impact of sphingomyelin synthase 1 deficiency on sphingolipid metabolism and atherosclerosis in mice. Arterioscler. Thromb. Vasc. Biol. 32: 1577-84 [PMID:22580896]

22. Li Z, Zhang H, Liu J, Liang CP, Li Y, Li Y, Teitelman G, Beyer T, Bui HH and Peake DAt al.. (2011) Reducing plasma membrane sphingomyelin increases insulin sensitivity. Mol. Cell. Biol. 31: 4205-18 [PMID:21844222]

23. Liu J, Huan C, Chakraborty M, Zhang H, Lu D, Kuo MS, Cao G and Jiang XC. (2009) Macrophage sphingomyelin synthase 2 deficiency decreases atherosclerosis in mice. Circ. Res. 105: 295-303 [PMID:19590047]

24. Mao C, Xu R, Szulc ZM, Bielawska A, Galadari SH and Obeid LM. (2001) Cloning and characterization of a novel human alkaline ceramidase. A mammalian enzyme that hydrolyzes phytoceramide. J. Biol. Chem. 276: 26577-88 [PMID:11356846]

25. Mao C, Xu R, Szulc ZM, Bielawski J, Becker KP, Bielawska A, Galadari SH, Hu W and Obeid LM. (2003) Cloning and characterization of a mouse endoplasmic reticulum alkaline ceramidase: an enzyme that preferentially regulates metabolism of very long chain ceramides. J. Biol. Chem. 278: 31184-91 [PMID:12783875]

26. Miyake Y, Kozutsumi Y, Nakamura S, Fujita T and Kawasaki T. (1995) Serine palmitoyltransferase is the primary target of a sphingosine-like immunosuppressant, ISP-1/myriocin. Biochem. Biophys. Res. Commun. 211: 396-403 [PMID:7794249]

27. Mizutani Y, Kihara A and Igarashi Y. (2005) Mammalian Lass6 and its related family members regulate synthesis of specific ceramides. Biochem. J. 390: 263-71 [PMID:15823095]

28. Mlinar B and Corradetti R. (2003) Endogenous 5-HT, released by MDMA through serotonin transporterand secretory vesicle-dependent mechanisms, reduces hippocampal excitatory synaptic transmission by preferential activation of 5-HT1B receptors located on CA1 pyramidal neurons. Eur. J. Neurosci. 18: 155971 [PMID:14511335]

29. Mo M, Yang J, Jiang XC, Cao Y, Fei J, Chen Y, Qi X, Chu Y, Zhou L and Ye D. (2018) Discovery of 4Benzyloxybenzo[ d]isoxazole-3-amine Derivatives as Highly Selective and Orally Efficacious Human Sphingomyelin Synthase 2 Inhibitors that Reduce Chronic Inflammation in db/ db Mice. J. Med. Chem. 61: 8241-8254 [PMID:30074791]

30. Philipp S, Puchert M, Adam-Klages S, Tchikov V, Winoto-Morbach S, Mathieu S, Deerberg A, Kolker L, Marchesini N and Kabelitz D et al.. (2010) The Polycomb group protein EED couples TNF receptor 1 to neutral sphingomyelinase. Proc. Natl. Acad. Sci. U.S.A. 107: 1112-7 [PMID:20080539]

31. Rabionet $M$, van der Spoel $A C$, Chuang $C C$, von Tümpling-Radosta $B$, Litjens $M$, Bouwmeester $D$, Hellbusch CC, Körner C, Wiegandt H and Gorgas K et al.. (2008) Male germ cells require polyenoic sphingolipids with complex glycosylation for completion of meiosis: a link to ceramide synthase-3. J. Biol. Chem. 283: 13357-69 [PMID:18308723]

32. Riebeling C, Allegood JC, Wang E, Merrill Jr AH and Futerman AH. (2003) Two mammalian longevity assurance gene (LAG1) family members, trh1 and trh4, regulate dihydroceramide synthesis using different fatty acyl-CoA donors. J. Biol. Chem. 278: 43452-9 [PMID:12912983]

33. Sun W, Xu R, Hu W, Jin J, Crellin HA, Bielawski J, Szulc ZM, Thiers BH, Obeid LM and Mao C. (2008) Upregulation of the human alkaline ceramidase 1 and acid ceramidase mediates calcium-induced differentiation of epidermal keratinocytes. J. Invest. Dermatol. 128: 389-97 [PMID:17713573]

34. Tani M, lida H and Ito M. (2003) O-glycosylation of mucin-like domain retains the neutral ceramidase on the plasma membranes as a type II integral membrane protein. J. Biol. Chem. 278: 10523-30 [PMID:12499379]

35. Tani M and Kuge O. (2009) Sphingomyelin synthase 2 is palmitoylated at the $\mathrm{COOH}$-terminal tail, which is involved in its localization in plasma membranes. Biochem. Biophys. Res. Commun. 381: 328-32 [PMID:19233134]

36. Venkataraman K, Riebeling C, Bodennec J, Riezman H, Allegood JC, Sullards MC, Merrill Jr AH and Futerman AH. (2002) Upstream of growth and differentiation factor 1 (uog1), a mammalian homolog of the yeast longevity assurance gene 1 (LAG1), regulates $\mathrm{N}$-stearoyl-sphinganine (C18-(dihydro)ceramide) synthesis in a fumonisin B1-independent manner in mammalian cells. J. Biol. Chem. 277: 35642-9 [PMID:12105227]

37. Wang K, Xu R, Snider AJ, Schrandt J, Li Y, Bialkowska AB, Li M, Zhou J, Hannun YA and Obeid LMet al.. 
(2016) Alkaline ceramidase 3 deficiency aggravates colitis and colitis-associated tumorigenesis in mice by hyperactivating the innate immune system. Cell Death Dis 7: e2124 [PMID:26938296]

38. Xu R, Jin J, Hu W, Sun W, Bielawski J, Szulc Z, Taha T, Obeid LM and Mao C. (2006) Golgi alkaline ceramidase regulates cell proliferation and survival by controlling levels of sphingosine and S1P. FASEB J. 20: 1813-25 [PMID:16940153]

39. Yang K, Nong K, Gu Q, Dong J and Wang J. (2018) Discovery of N-hydroxy-3-alkoxybenzamides as direct acid sphingomyelinase inhibitors using a ligand-based pharmacophore model. Eur J Med Chem 151: 389400 [PMID:29649738]

40. Yang K, Yu J, Nong K, Wang Y, Niu A, Chen W, Dong J and Wang J. (2020) Discovery of Potent, Selective, and Direct Acid Sphingomyelinase Inhibitors with Antidepressant Activity. J. Med. Chem. 63: 961-974 [PMID:31944697]

41. Yokomatsu T, Murano T, Akiyama T, Koizumi J, Shibuya S, Tsuji Y, Soeda S and Shimeno H. (2003) Synthesis of non-competitive inhibitors of sphingomyelinases with significant activity. Bioorg. Med. Chem. Lett. 13: 229-36 [PMID:12482429]

42. Young SA, Mina JG, Denny PW and Smith TK. (2012) Sphingolipid and ceramide homeostasis: potential therapeutic targets. Biochem Res Int 2012: 248135 [PMID:22400113] 